\title{
Unconventional orbital charge density wave mechanism in the transition metal dichalcogenide $1 T-\mathrm{TaS}_{2}$
}

\author{
Toru Hirata, Youichi Yamakawa, Seiichiro Onari $\odot$, and Hiroshi Kontani $\odot$ \\ Department of Physics, Nagoya University, Furo-cho, Nagoya 464-8602, Japan
}

(Received 27 November 2020; revised 9 June 2021; accepted 2 July 2021; published 27 August 2021)

\begin{abstract}
The transition metal dichalcogenide $1 T-\mathrm{TaS}_{2}$ is attracting growing attention because of the formation of rich density wave (DW) and superconducting transitions. However, the origin of the incommensurate DW state at the highest temperature $(\sim 550 \mathrm{~K})$, which is "the parent state" of the rich physical phenomena, is still uncovered. Here, we present a natural explanation for the triple- $\boldsymbol{q}$ incommensurate DW in $1 T-\mathrm{TaS}_{2}$ based on the firstprinciples Hubbard model with on-site $U$. We apply the paramagnon interference mechanism that gives the nematic order in Fe-based superconductors. The derived order parameter has very unique characters: (i) an orbital-selective nature, and (ii) an unconventional sign reversal in both momentum and energy spaces. The present Letter will be useful for understanding the rich physics in $1 T-\mathrm{TaS}_{2}, 1 T-\mathrm{VSe}_{2}$, and other transition metal dichalcogenides.
\end{abstract}

DOI: 10.1103/PhysRevResearch.3.L032053

Transition metal dichalcogenides (TMDs) provide a promising platform for exotic low-dimensional electronic states with strong electron correlation. Among the TMDs, $1 T$ $\mathrm{Ta}(\mathrm{S}, \mathrm{Se})_{2}$ exhibits very interesting electronic properties, such as a metal-insulator transition with a star-of-David formation as well as exotic superconductivity. The electronic states are easily controlled by gate electric field carrier doping [1-3], by changing the dimensionality [4-6], and by applying pressure $[7,8]$ and picosecond laser pulses [9].

In $1 T-\mathrm{TaS}_{2}$, at ambient pressure, an incommensurate charge density wave (IC-CDW) appears as the highest transition temperature at $T=T_{\mathrm{IC}} \approx 550 \mathrm{~K}$ [10]. With decreasing $T$, the IC-CDW changes to a nearly commensurate (NC) CDW at $T_{\mathrm{NC}} \approx 350 \mathrm{~K}$, and finally a star-of-David commensurate $(\mathrm{C}) \mathrm{CDW}$ appears at $T_{\mathrm{C}} \approx 200 \mathrm{~K}$ successively. This rich multistage $\mathrm{CDW}$ transition is suppressed under pressure, and superconductivity emerges at $T_{\mathrm{SC}} \lesssim 10 \mathrm{~K}$. These exotic ordered states emerge under the IC-CDW state. That is, the IC-CDW is the parent electronic state of the rich physics in $1 T-\mathrm{Ta}(\mathrm{S}, \mathrm{Se})_{2}$ [11-19]. Nonetheless, the understanding of the origin and nature of the IC-CDW is very limited at present.

Although phonon-driven CDW inevitably causes a sizable lattice distortion (LD) in proportion to the transition temperature, the LD below $T_{\mathrm{IC}}$ is much smaller than that below $T_{\mathrm{C}}$ in $1 T-\mathrm{TaS}_{2}$ [20,21]. Considering the importance of the electron correlation in $1 T-\mathrm{TaS}_{2}$, it is important to investigate the electron-correlation-driven IC-CDW mechanism, although the derivation of "nonmagnetic IC-CDW order" is a very difficult theoretical problem. In fact, magnetic order

Published by the American Physical Society under the terms of the Creative Commons Attribution 4.0 International license. Further distribution of this work must maintain attribution to the author(s) and the published article's title, journal citation, and DOI. is always obtained based on Hubbard models with on-site $U$ within mean-field theories. Thus, one may consider the existence of large off-site bare interactions [such as the off-site Coulomb interaction and Ruderman-Kittel-KasuyaYosida (RKKY) interaction [22]] comparable to $U$. Therefore, the study of IC-CDW is very important to uncover the real Hamiltonian for $1 T-\mathrm{TaS}_{2}$, based on which multistage CDW transition and superconductivity should be studied.

Recently, in various strongly correlated metals, electronic nematic states have been actively studied by using beyondmean-field theories [23-39], especially the paramagnoninterference mechanism of density wave orders [26-39]. Although the IC-CDW state in $1 T-\mathrm{TaS}_{2}$ is not nematic, it is a promising challenge to apply the paramagnon-interference mechanism to this long-standing problem.

In this Letter, we present a natural explanation for high-

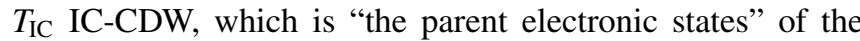
exotic multistage CDW and superconductivity in $1 T-\mathrm{TaS}_{2}$. The predicted IC-CDW is the correlation-driven "unconventional CDW," in which the CDW order parameter possesses strange orbital momentum energy dependences, in analogy to the unconventional superconductivity. The wave vectors of the IC-CDW state coincide with the Fermi surface (FS) nesting vectors $\boldsymbol{q}=\boldsymbol{q}_{1}, \boldsymbol{q}_{2}, \boldsymbol{q}_{3}$ in Fig. 1(a) [40,41]. In addition, with the aid of the Ginzburg-Landau (GL) theory, we reveal that the triple- $\boldsymbol{q}$ CDW state is stabilized. This Letter provides the necessary knowledge to resolve the mysterious C-CDW state [42-49]. This theory will be useful for understanding rich CDW states in $1 T-\mathrm{TaS}_{2}, 1 T-\mathrm{VSe}_{2}$, and other TMDs.

First, we construct the first-principles 11-orbital tightbinding model of $1 T-\mathrm{TaS}_{2}, H_{0}$, composed of five $5 d^{1}$ orbitals of Ta ions and six $3 p$ orbitals of $\mathrm{S}$ ions, using the WIEN2K and WANNIER90 software. The $d$-electron eigenfunctions in the $\mathrm{S}_{6}$ octahedron under the trigonal distortion are composed of one $a_{1 g}$ orbital, two $e_{g}^{\prime}$ orbitals, and two $e_{g}$ orbitals. In this Letter, we assign $a_{1 g}, e_{g}^{\prime}$, and $e_{g}$ orbitals as orbitals $1,(2,3)$, and 

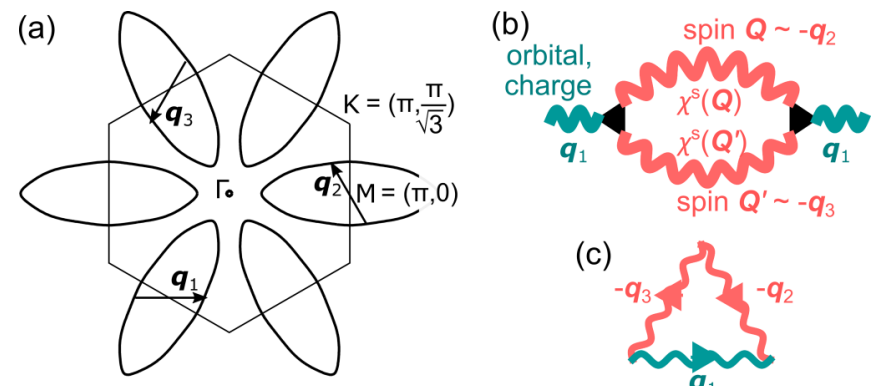

(c)

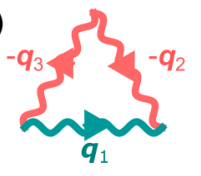

FIG. 1. (a) Three electron-type FSs and the intra-FS nesting vectors $\boldsymbol{q}_{n}(n=1,2,3)$. Here, $\boldsymbol{q}_{1}=(0.56 \pi, 0)$. (b) Paramagnon interference mechanism of charge/orbital order at wave vector $\boldsymbol{q}_{1}$. (c) Momentum conservation law in the interference mechanism.

$(4,5)$ in order. The FSs are mainly composed of orbitals 1-3. The wave functions of orbitals $1-5$ and the model Hamiltonian based on an experimental crystal structure [50] are given in the Supplemental Material (SM) A [51].

We also introduce the on-site Coulomb interaction Hamiltonian $H_{U}$. It is composed of the intraorbital (interorbital) interaction $U\left(U^{\prime}\right)$, and the exchange interaction $J=(U-$ $\left.U^{\prime}\right) / 2$. Below, we fix the ratio $J / U=0.1$. (The obtained results are similar for $J / U<0.2$.) These interactions are included into the spin (charge) channel interaction matrix $\hat{\Gamma}^{s(c)}$; see SM A [51] for details.

Here, we calculate the spin susceptibility using the random phase approximation (RPA), $\hat{\chi}^{s}(q)=\hat{\chi}^{0}(q)\left[\hat{1}-\hat{\Gamma}^{s} \hat{\chi}^{0}(q)\right]^{-1}$, where $q \equiv\left(\boldsymbol{q}, \omega_{l}=2 \pi T l\right)$. Here, $\hat{\chi}^{0}(q)$ is the irreducible susceptibility matrix in SM B [51]. The spin Stoner factor $\alpha_{S}$ is the maximum eigenvalue of $\hat{\Gamma}^{s} \hat{\chi}^{0}(q)$. The relation $\hat{\chi}^{s}(\boldsymbol{q}) \propto$ $\left(1-\alpha_{S}\right)^{-1}$ is satisfied when $\boldsymbol{q}$ is the nesting vector, and the magnetic order appears when $\alpha_{S}=1$. The total spin susceptibility $\chi_{\text {tot }}^{s}(\boldsymbol{q})=\sum_{l, m=1}^{5} \chi_{l, l ; m, m}^{s}(\boldsymbol{q})$ exhibits broad peaks at $\boldsymbol{q} \approx \boldsymbol{q}_{i}(i=1-3)$, as we show in Fig. S2(a) in SM B [51]. The components $\chi_{l, m ; l, m}^{s}(\boldsymbol{q})$ with $1 \leqslant l, m \leqslant 3$ are large as shown in Fig. S2, because the orbitals 1-3 are heavily entangled on the FSs, as shown in Fig. S1.

However, the IC-CDW without magnetization cannot be explained by the RPA because the charge Stoner factor is always smaller than $\alpha_{S}$ in the RPA [26]. To explain the IC-CDW state, we study the charge-channel susceptibility $\chi^{\mathrm{DW}}(\boldsymbol{q})$ due to the higher-order vertex corrections (VCs), based on the density wave (DW) equation method [27,33]. (The VCs are dropped in the RPA.) Figure 1(b) is an Aslamazov-Larkin (AL) type VC for $\chi^{\mathrm{DW}}(\boldsymbol{q})$, which is proportional to the convolution of paramagnons $C_{\boldsymbol{q}} \sim \sum_{\boldsymbol{p}} \chi^{s}\left(\boldsymbol{q}_{1}+\boldsymbol{p}\right) \chi^{s}(\boldsymbol{p})$. We will show that the AL type VC induces the IC-CDW order at $\boldsymbol{q}=\boldsymbol{q}_{i}(i=1,2,3)$, since $C_{\boldsymbol{q}}$ is large at $\boldsymbol{q}=\boldsymbol{q}_{i}$ due to the momentum conservation in Fig. 1(c).

Here, we introduce the linearized charge-channel DW equation $[27,33]$,

$$
\begin{aligned}
\lambda_{\boldsymbol{q}} f_{\boldsymbol{q}}^{L}(k)= & -\frac{T}{N} \sum_{p, M_{1}, M_{2}} I_{\boldsymbol{q}}^{L, M_{1}}(k, p) \\
& \times\left\{G\left(p_{-}\right) G\left(p_{+}\right)\right\}^{M_{1}, M_{2}} f_{\boldsymbol{q}}^{M_{2}}(p),
\end{aligned}
$$

where $\boldsymbol{k}_{ \pm} \equiv \boldsymbol{k} \pm \boldsymbol{q} / 2, k \equiv\left(\boldsymbol{k}, \epsilon_{n}\right)$, and $p \equiv\left(\boldsymbol{p}, \epsilon_{m}\right)\left(\epsilon_{n}, \epsilon_{m}\right.$ are fermion Matsubara frequencies). $L \equiv\left(l, l^{\prime}\right)$ and $M_{i}$
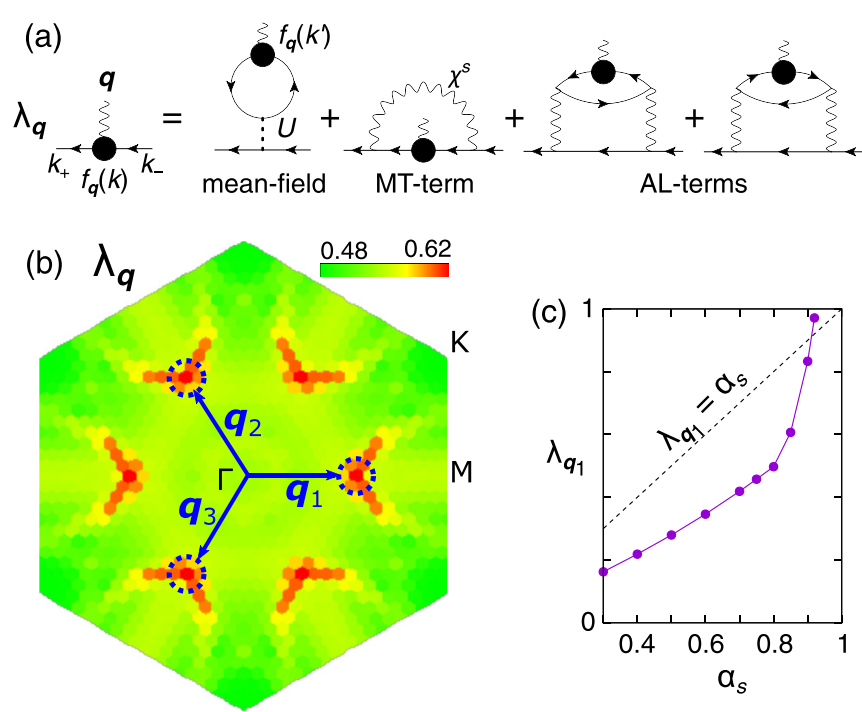

FIG. 2. (a) Diagrammatic expression of the DW equation. (b) Obtained eigenvalue of the DW equation $\lambda_{q}$ for $T=40 \mathrm{meV}$ and $\alpha_{S}=0.85 . \lambda_{\boldsymbol{q}}$ shows the peaks at the intra-FS nesting vectors $q=\boldsymbol{q}_{n}$ $(n=1,2,3)$, consistently with experiments. Here, $\boldsymbol{q}_{1}=(0.56 \pi, 0)$. (c) Eigenvalue $\lambda_{\boldsymbol{q}_{n}}$ as a function of $\alpha_{S}$. It reaches unity for $\alpha_{S} \gtrsim 0.90$.

represents the pair of $d$-orbital indices. $\lambda_{q}$ is the eigenvalue and $f_{\boldsymbol{q}}^{L}(k)$ is the Hermite form factor. The former represents the instability of the DW fluctuations at wave vector $\boldsymbol{q}$, which reaches unity when long-range order is established. The latter is the general charge-channel order parameter: $f_{\boldsymbol{q}}^{l, l^{\prime}}(\boldsymbol{k})=$ $\sum_{\sigma}\left\langle c_{\boldsymbol{k}_{+}, l, \sigma}^{\dagger} c_{\boldsymbol{k}_{-}, l^{\prime}, \sigma}\right\rangle-\left\langle c_{\boldsymbol{k}_{+}, l, \sigma}^{\dagger} c_{\boldsymbol{k}_{-}, l^{\prime}, \sigma}\right\rangle_{0}$. The DW equation (1) is interpreted as the "charge-channel electron-hole pairing equation" with the pairing interaction $I_{q}^{L, M}(k, p)$.

$I_{\boldsymbol{q}}^{L, M}$ at $\boldsymbol{q}=\mathbf{0}$ is given by the Ward identity $-\delta \Sigma^{L}(k) / \delta G^{M}\left(k^{\prime}\right)$ that is composed of one single-magnon exchange Maki-Thompson (MT) term and two doublemagnon interference AL terms; see Fig. 2(a). Here, we set $T=0.04 \mathrm{eV}$ and $U=3.87 \mathrm{eV} \quad\left(\alpha_{S}=0.85\right)$. [Since $d$-electron weight in the density of states (DOS) at the Fermi level is about $70 \%, U$ is reduced to $\sim 2.9 \mathrm{eV}$ in the $d$-orbital Hubbard model.] The analytic expressions of the MT and AL terms are explained in SM C [51]. The AL terms are proportional to the convolution of paramagnons $C_{\boldsymbol{q}}$ so they become important when $\alpha_{S}$ approaches unity [26,33]. Their essential role has been revealed by the functional renormalization group (fRG) study in which higher-order VCs are produced in an unbiased way [30,32]. In contrast, the MT term is important for the superconducting gap equation and for the transport phenomena [52].

Figure 2(b) shows the obtained eigenvalue of the DW equation $\lambda_{q}$ for $T=0.04 \mathrm{eV}$ and $\alpha_{S}=0.85$; we see that $\lambda_{q}$ has six peaks at the nesting vector $\left(\boldsymbol{q}= \pm \boldsymbol{q}_{n}, n=1,2,3\right)$. As shown in Fig. 2(c), the eigenvalue $\lambda_{q_{1}}$ increases with $U$, and it exceeds $\alpha_{S}$ and reaches unity for $\alpha_{S} \gtrsim 0.90$. The susceptibility of the DW is given as $\chi^{\mathrm{DW}}(\boldsymbol{q}) \propto 1 /\left(1-\lambda_{\boldsymbol{q}}\right)$. Thus, the obtained results are consistent with the IC-CDW order at $\boldsymbol{q}= \pm \boldsymbol{q}_{n}$ without magnetization in $1 T-\mathrm{TaS}_{2}$.

We stress that $T_{\mathrm{IC}}$ is enlarged by phonons in the case where the form factor of the electron-phonon interaction is 

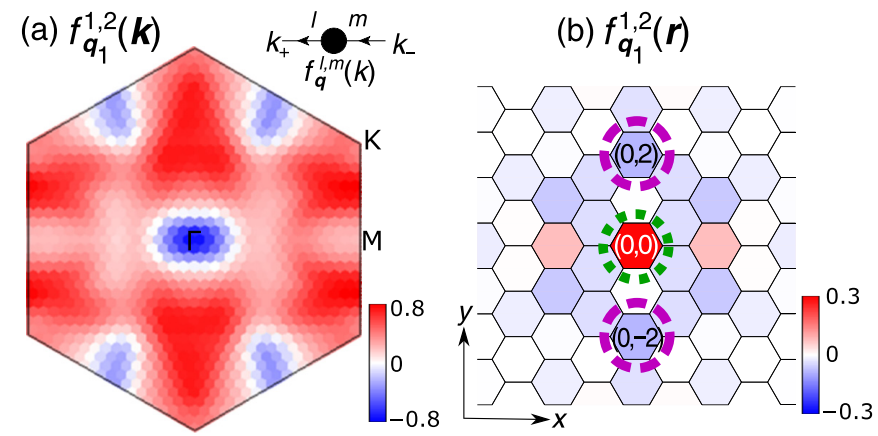

FIG. 3. (a) Real part of obtained form factor $f_{q_{1}}^{1,2}(\boldsymbol{k})$, which is the most significant component for the IC-CDW at $\boldsymbol{q}=\boldsymbol{q}_{1}$. (b) Fourier transformation of the form factor $f_{q_{1}}^{1,2}(\boldsymbol{r})$. The signal at $\boldsymbol{r}=\mathbf{0}$ represents the local orbital+charge order with respect to orbitals 1 and 2 , and the signal at $\boldsymbol{r} \neq \mathbf{0}$ exhibits the nonlocal bond order. The strongest bond order is at $\boldsymbol{r}=(0, \pm 2)$ marked by pink dashed circles.

equal to the DW form factor in symmetry [53]. As discussed in Ref. [53], the "total DW susceptibility" is given as $\chi_{\mathrm{tot}}^{\mathrm{DW}}(\boldsymbol{q})=\chi^{\mathrm{DW}}(\boldsymbol{q}) /\left[1-2 g \chi^{\mathrm{DW}}(\boldsymbol{q})\right]$, where $g(>0)$ is the phonon-induced attraction. In fact, in Fe-based superconductors, the nematic transition temperature is raised by $\sim 50 \mathrm{~K}$ due to the $B_{1 g}$ phonon mode. A similar phonon-assisted increment of $T_{\mathrm{IC}}$ is expected in $1 T-\mathrm{TaS}_{2}$.

Here, we discuss the nature of the form factor at $\boldsymbol{q}=\boldsymbol{q}_{i}$. In Fig. 3(a), we show the obtained real parts of $f_{\boldsymbol{q}_{1}}^{1,2}(\boldsymbol{k})$ at the lowest Matsubara frequency. It is the largest intraorbital component of the form factor $\hat{f}_{\boldsymbol{q}_{1}}(\boldsymbol{k})$. The largest intraorbital component is $f_{\boldsymbol{q}_{1}}^{1,1}(\boldsymbol{k})$, which is exhibited in Fig. S3(a) in SM B [51]. Other important form factors $f_{\boldsymbol{q}_{1}}^{l, m}(\boldsymbol{k})$ with $1 \leqslant l, m \leqslant 3$ are shown in Figs. S3(b)-S3(e). The parity of the obtained order $(\boldsymbol{k} \leftrightarrow-\boldsymbol{k})$ is even. The off-diagonal component $f_{\boldsymbol{q}_{1}}^{1,2}$ induces the orbital polarization; $\left(|1\rangle, E_{1}\right) \rightarrow\left(|1\rangle+c|2\rangle, E_{1}+\right.$ $\Delta E)$ and $\left(|2\rangle, E_{2}\right) \rightarrow\left(|2\rangle-c|1\rangle, E_{2}-\Delta E\right)$ with $|c| \leqslant 1$. It also induces the finite charge order $(\delta n)$ since $E_{1} \neq E_{2}$ in the present model, as shown Fig. S1(b) in SM A [51]. Interestingly, Fig. 3(a) has sign reversal, which is very different from the conventional CDW with a nearly constant form factor. A similar "sign reversing form factor" is observed in the nematic phase in FeSe by angle-resolved photoemission spectroscopy (ARPES) measurement [54], and it is satisfactorily explained as the paramagnon interference mechanism [27].

Next, we examine the real-space DW structure with $\boldsymbol{q}=$ $\boldsymbol{q}_{n}$. For this purpose, we perform the Fourier transformation of the form factor,

$$
\begin{gathered}
\tilde{f}^{l, m}\left(\boldsymbol{r}_{i}, \boldsymbol{r}_{j}\right)=\operatorname{Re}\left\{f_{\boldsymbol{q}_{n}}^{l, m}\left(\boldsymbol{r}_{i}-\boldsymbol{r}_{j}\right) e^{i \boldsymbol{q}_{n} \cdot\left(\boldsymbol{r}_{i}+\boldsymbol{r}_{j}\right) / 2+i \theta}\right\}, \\
f_{\boldsymbol{q}_{n}}^{l, m}(\boldsymbol{r})=\frac{1}{N} \sum_{\boldsymbol{k}} f_{\boldsymbol{q}_{n}}^{l, m}(\boldsymbol{k}) e^{i \boldsymbol{r} \cdot \boldsymbol{k}},
\end{gathered}
$$

where $\boldsymbol{r}_{i}$ is the real-space position of site $i$, and $\theta$ is the arbitrary phase factor. Here, $\tilde{f}^{l, m}\left(\boldsymbol{r}_{i}, \boldsymbol{r}_{i}\right)$ represents the local charge and/or orbital order at $\boldsymbol{r}_{i}$, and $\tilde{f}^{l, m}\left(\boldsymbol{r}_{i}, \boldsymbol{r}_{j}\right)$ with $i \neq j$ is the bond order (i.e., the modulation of the hopping integral) between $\boldsymbol{r}_{i}$ and $\boldsymbol{r}_{j}$. Figure 3(b) is the obtained $f_{\boldsymbol{q}_{1}}^{1,2}(\boldsymbol{r})$ : Its large magnitude at $\boldsymbol{r}=\mathbf{0}$ represents the orbital order with respect to $|1\rangle \pm|2\rangle$. In addition, $f_{\boldsymbol{q}_{1}}^{1,2}(\boldsymbol{r})$ shows large values for $\boldsymbol{r} \neq \mathbf{0}$. Thus, both orbital order and bond order coexist
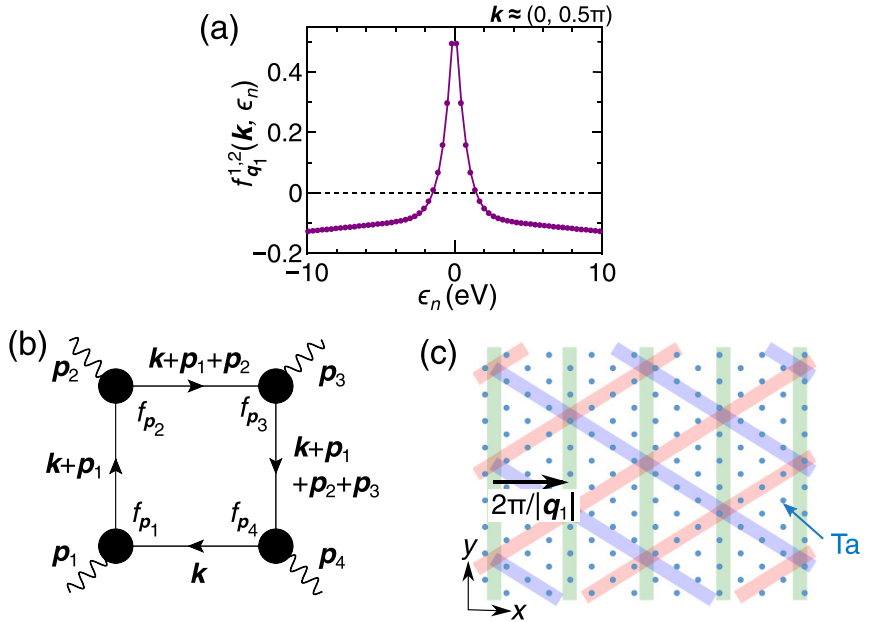

FIG. 4. (a) $\operatorname{Re} f_{q_{1}}^{1,2}\left(\boldsymbol{k}, \epsilon_{n}\right)$ as a function of $\epsilon_{n}$ at $\boldsymbol{k}=(0,0.5 \pi)$. (b) Diagrammatic expression of the fourth-order term in the GL equation. (c) Triple- $\boldsymbol{q}$ IC-CDW state in real space.

in the obtained IC-CDW state. The trace of the form factor, $f_{\boldsymbol{q}_{1}}^{\mathrm{tr}}(\boldsymbol{r}) \equiv \sum_{l} f_{\boldsymbol{q}_{1}}^{l, l}(\boldsymbol{r})$, is shown in Fig. S3(f) in SM B. The large value of $f_{\boldsymbol{q}_{1}}^{\text {tr }}(\boldsymbol{r})$ at $\boldsymbol{r}=\mathbf{0}$ means the emergence of the local charge order. To summarize, the IC-CDW in $1 T-\mathrm{TaS}_{2}$ is identified as a combination of the charge/orbital/bond order in the present study.

In the obtained IC-CDW state, essentially all elements of the form factor $f_{\boldsymbol{q}_{n}}^{l, m}$ in the $a_{1 g}+e_{g}^{\prime}$ orbital space $(l, m=1-3)$ are large. This fact indicates that all $a_{1 g}+e_{g}^{\prime}$ orbital states cooperatively magnify the eigenvalue. In order to identify the major order parameter, we solve the DW equation (1) for $\boldsymbol{q}=\boldsymbol{q}_{1}$ by considering only $f_{\boldsymbol{q}_{1}}^{1,2}$ and $f_{\boldsymbol{q}_{1}}^{2,1}$, by setting other elements zero. In this case, the obtained form factor $f_{\boldsymbol{q}_{1}}^{1,2}(\boldsymbol{k})$ is very similar to Fig. S3(a), and $\lambda_{q_{1}}$ is reduced just to $86 \%$ from Fig. 2(b). In contrast, the eigenvalue becomes very small if only diagonal elements $f_{\boldsymbol{q}_{1}}^{l, l}$ are considered. Therefore, the off-diagonal form factor $f_{\boldsymbol{q}_{1}}^{1,2}$ is the main order parameter of the IC-CDW state.

In the present mechanism, the orbital+charge DW order due to $f_{q_{1}}^{1,2} \neq 0$ originates from the interference among spin fluctuations at $\boldsymbol{q}=\boldsymbol{q}_{2}$ and $\boldsymbol{q}=\boldsymbol{q}_{3}$, as depicted in Fig. 1(c). Mathematically, $f_{q_{1}}^{1,2}$ is derived from the kernel $I_{\boldsymbol{q}_{1}}^{L, M}$. However, local net charge order $(\delta n)$ is energetically unfavorable due to the mean-field term $(\sim U \delta n)$ in the first term of Fig. 2(a). In fact, in Fe-based and cuprate superconductors, the bond and orbital orders with $\delta n=0$ appear since they are not prohibited by on-site $U[27,32]$. To understand why net charge order is obtained in the $1 T-\mathrm{TaS}_{2}$ model, we examine the energy dependence of the form factor. Figure 4(a) shows the frequency $\left(\epsilon_{n}\right)$ dependence of $\operatorname{Re} f_{\boldsymbol{q}_{1}}^{1,2}\left(\boldsymbol{k}, \epsilon_{n}\right)$ near the van Hove singular point $\boldsymbol{k}=(0,0.5 \pi)$. A similar sign reversal appears in other elements of the form factor. This sign reversing form factor is very similar to the sign reversing gap function in the $s$-wave superconductors with $U \neq 0$, known as the "retardation effect" that drastically reduces the depairing by $U$. Thus, net charge order in the IC-CDW state in $1 T-\mathrm{TaS}_{2}$, which is very unusual in metals with large $U$, is stabilized by the retardation effect. To summarize, the predicted "un- 
conventional CDW state" in $1 T-\mathrm{TaS}_{2}$ is characterized by the orbital selective form factor with strange sign reversals in the momentum and energy spaces.

Finally, we explain the "triple- $\boldsymbol{q}$ CDW state" in $1 T-\mathrm{TaS}_{2}$, which is the uniform coexisting state of the three order parameters with $\boldsymbol{q}=\boldsymbol{q}_{1}, \boldsymbol{q}_{2}, \boldsymbol{q}_{3}$. For this purpose, we construct a simple Ginzburg-Landau free energy for the CDW order $\vec{\Phi}(k)=\left[\eta_{1} f_{\boldsymbol{q}_{1}}(k), \eta_{2} f_{\boldsymbol{q}_{2}}(k), \eta_{2} f_{\boldsymbol{q}_{2}}(k)\right]$, where $\eta_{i}$ is the real order parameter and $f_{\boldsymbol{q}_{i}}(k)$ is the normalized form factor. Then, the free energy is given by $F=a_{0}\left(\left|\eta_{1}\right|^{2}+\left|\eta_{2}\right|^{2}+\left|\eta_{3}\right|^{2}\right)+b_{0}\left(\left|\eta_{1}\right|^{4}+\left|\eta_{2}\right|^{4}+\left|\eta_{3}\right|^{4}\right)+$ $c_{0}\left(\left|\eta_{1} \eta_{2}\right|^{2}+\left|\eta_{2} \eta_{3}\right|^{2}+\left|\eta_{3} \eta_{1}\right|^{2}\right)$, where $a_{0} \propto T-T_{\mathrm{IC}}$. The fourth-order coefficients $b_{0}$ and $c_{0}$ can be calculated by using the Green's functions and form factors; see Fig. 4(b). The derivation of $a_{0}, b_{0}$, and $c_{0}$ in addition to the third-order term $F^{(3)}=d_{0} \eta_{1} \eta_{2} \eta_{3}$ is given in SM D [51]. Here, the single- $\boldsymbol{q}$ state and the triple- $\boldsymbol{q}$ state correspond to $\left(\eta_{1}, \eta_{2}, \eta_{3}\right)=(\eta, 0,0)$ and $(\eta, \eta, \eta)$, respectively. It is easy to show that the triple- $\boldsymbol{q}$ condition is $c_{0} / b_{0}<2$ if $d_{0}$ is negligible. As we show in SM D [51], the ratio $c_{0} / b_{0}=1.1$ is obtained by using the form factors in the present study. $\left[f_{q_{1}}^{l m}\right.$ is given in Figs. 3(a) and S3.] Thus, the present IC-CDW state satisfies the triple- $\boldsymbol{q}$ condition. In contrast, in the case of a conventional CDW form factor with $f_{\boldsymbol{q}_{n}}^{l, m}=\delta_{l, m}$, the obtained ratio $c_{0} / b_{0}=3.2$ does not satisfy the triple- $\boldsymbol{q}$ condition; see SM D [51]. Thus, the obtained unconventional form factor due to the $\mathrm{AL}$ processes is indispensable to explain the triple- $\boldsymbol{q}$ IC-CDW state in $1 T-\mathrm{TaS}_{2}$, which is schematically shown in Fig. 4(c).

Here, we calculate the electronic states below $T_{\mathrm{IC}}$ based on the $4 \times 4$ cluster tight-binding model with finite DW order given in Eq. (2). We make the wave vector of the DW order $\boldsymbol{q}_{1}=(0.5 \pi, 0)$ by introducing $20 \%$ hole doping. Although the folded FS under the CDW state is very complex, it can be "unfolded" to the original Brillouin zone (BZ) by restoring the translational symmetry of the spectral function [55]. Figures 5(a) and 5(b) show the obtained unfolded FS under the single- $\boldsymbol{q}$ and triple- $\boldsymbol{q}$ CDW states, respectively, by setting $f^{\max } \equiv \max _{l, m, \boldsymbol{k}}\left\{f_{\boldsymbol{q}_{1}}^{l, m}(\boldsymbol{k})\right\}=88 \mathrm{meV}$. In the single- $\boldsymbol{q}_{1}$ case, a sizable Fermi arc appears in FS1,3 due to the band folding by $\hat{f}_{q_{1}}$. In the same way, Fermi arc appears in FS1,2 (FS2,3) by $\hat{f}_{\boldsymbol{q}_{2}}\left(\hat{f}_{\boldsymbol{q}_{3}}\right)$. The expected charge density modulation by $\left|f_{\boldsymbol{q}_{1}}^{\mathrm{tr}}(\boldsymbol{r}=\mathbf{0})\right| \sim 0.5$ in Fig. S3(f) is $\delta n \sim\left[f^{\mathrm{max}} \mid f_{\boldsymbol{q}_{1}}^{\text {tr }}(\boldsymbol{r}=\right.$ 0) $\mid] N(0) \stackrel{\sim}{\sim} 0.02$.

Counterintuitively, the size of the Fermi arc in the triple$q$ case in Fig. 5(b) is much reduced, where we set $f^{\max }=$ $(88 / \sqrt{2})$ meV because $b_{0} \approx c_{0}$ in the present study; see SM D [51]. To understand the reason, we consider the hybridization between Fermi momenta $\boldsymbol{k}, \boldsymbol{k}+\boldsymbol{q}_{1}$, and $\boldsymbol{k}-\boldsymbol{q}_{2}$ in Fig. 5(c). In the FS reconstruction by two form factors $\hat{f}_{\boldsymbol{q}_{1}}$ and $\hat{f}_{\boldsymbol{q}_{2}}$, the state $|\boldsymbol{k}\rangle$ hybridizes with $\left|\boldsymbol{k}+\boldsymbol{q}_{1}\right\rangle$ and $\left|\boldsymbol{k}-\boldsymbol{q}_{2}\right\rangle$ at the same time. Since $\hat{f}_{\boldsymbol{q}_{1}} \sim \hat{f}_{\boldsymbol{q}_{2}}$, one eigenstate $\left|\boldsymbol{k}+\boldsymbol{q}_{1}\right\rangle-\mid \boldsymbol{k}-$ $\left.\boldsymbol{q}_{2}\right\rangle$ is unhybridized and therefore gapless. (For general hybridization potentials, one of the three bands always remains
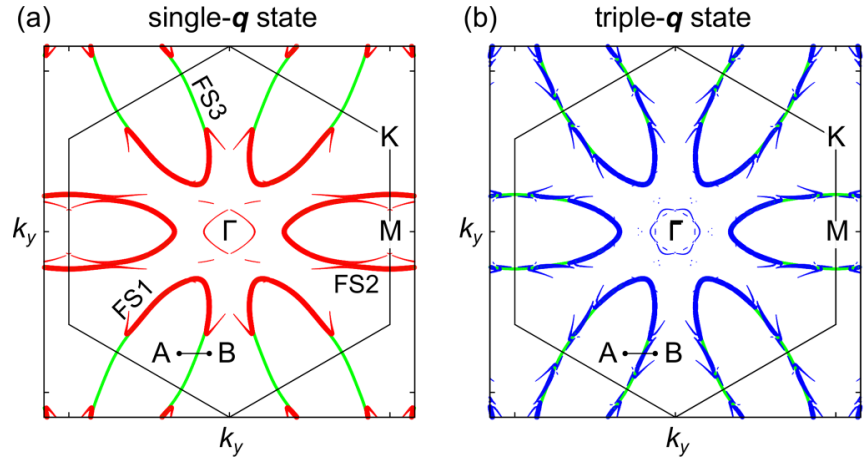

(c)
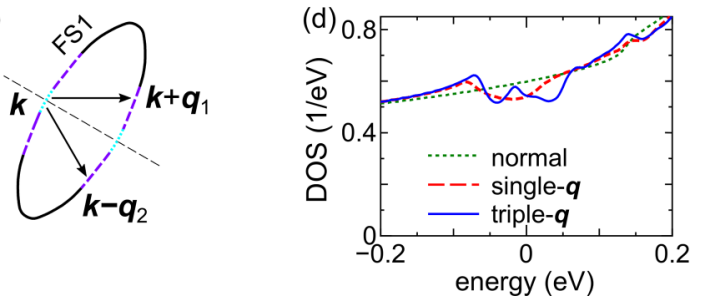

FIG. 5. (c) Unfolded FS in the single- $\boldsymbol{q}_{1}$ state and (d) that in the triple- $\boldsymbol{q}$ state. (c) Reason for the FS recovery in the triple- $\boldsymbol{q}$ CDW, which occurs when the state $|\boldsymbol{k}\rangle$ hybridizes with $\left|\boldsymbol{k}+\boldsymbol{q}_{1}\right\rangle$ and $\left|\boldsymbol{k}-\boldsymbol{q}_{2}\right\rangle$ simultaneously. (d) DOS as a function of $\epsilon-E_{\mathrm{F}}$. We introduced a BCS-type cutoff $\omega_{c}=4 f^{\max }$.

unhybridized.) For this reason, after the unfolding, the Fermi arc structure around $\boldsymbol{k}+\boldsymbol{q}_{1}$ and $\boldsymbol{k}-\boldsymbol{q}_{2}$ in Fig. 5(a) is recovered, as shown in Fig. 5(b). Also, the spectral recovery in the unfolded band structure is explained in SM B [51]. This hallmark in the triple- $\boldsymbol{q}$ CDW state could be observed by a high-resolution ARPES study. Figure 5(d) shows the obtained density of states (DOS). The pseudogap at $E_{\mathrm{F}}$ in the triple- $q$ CDW is small by reflecting the short Fermi arc in Fig. 5(b). This result is consistent with the experimental good metallic state below $T_{\mathrm{IC}}$.

In summary, we succeeded in explaining the triple- $q$ IC$\mathrm{CDW}$ in $1 T-\mathrm{TaS}_{2}$ in terms of the "unconventional CDW," in which the form factor has strange orbital momentum energy dependences. Owing to the present paramagnon interference mechanism, the triple- $\boldsymbol{q}$ IC-CDW state is naturally understood based on a simple Hubbard model with on-site $U$, without introducing any nonlocal interactions. The same mechanism would be applicable for $1 T-\mathrm{VSe}_{2}$ and other TMDs. Based on the knowledge on the IC-CDW state obtained by this study, it would be useful to develop a Ginzburg-Landau theory to understand the NC- and C-CDW states.

We are grateful to R. Tazai for useful discussions. This work was supported by the "Quantum Liquid Crystals" No. JP19H05825 KAKENHI on Innovative Areas from JSPS of Japan, and JSPS KAKENHI (JP18H01175, JP17K05543, JP20K03858).
[1] Y. Yu, F. Yang, X. F. Lu, Y. J. Yan, Y.-H. Cho, L. Ma, X. Niu, S. Kim, Y.-W. Son, D. Feng, S. Li, S.-W. Cheong, X. H. Chen, and Y. Zhang, Gate-tunable phase transitions in thin flakes of $1 \mathrm{~T}-\mathrm{TaS}_{2}$, Nat. Nanotechnol. 10, 270 (2015). 
[2] L. J. Li, E. C. T. O’Farrell, K. P. Loh, G. Eda, B. Özyilmaz, and A. H. Castro Neto, Controlling many-body states by the electric-field effect in a two-dimensional material, Nature (London) 529, 185 (2016).

[3] M. Yoshida, R. Suzuki, Y. Zhang, M. Nakano, and Y. Iwasa, Memristive phase switching in two-dimensional $1 \mathrm{~T}-\mathrm{TaS}_{2}$ crystals, Sci. Adv. 1, e1500606 (2015).

[4] A. W. Tsen, R. Hovden, D. Wang, Y. D. Kim, J. Okamoto, K. A. Spoth, Y. Liu, W. Lu, Y. Sun, J. C. Hone, L. F. Kourkoutis, P. Kim, and A. N. Pasupathy, Structure and control of charge density waves in two-dimensional $1 \mathrm{~T}-\mathrm{TaS}_{2}$, Proc. Natl. Acad. Sci. U.S.A. 112, 15054 (2015).

[5] P. C. Börner, M. K. Kinyanjui, T. Björkman, T. Lehnert, A. V. Krasheninnikov, and U. Kaiser, Observation of charge density waves in free-standing 1T-TaSe 2 monolayers by transmission electron microscopy, Appl. Phys. Lett. 113, 173103 (2018).

[6] Y. D. Wang, W. L. Yao, Z. M. Xin, T. T. Han, Z. G. Wang, L. Chen, C. Cai, Y. Li, and Y. Zhang, Band insulator to Mott insulator transition in $1 \mathrm{~T}-\mathrm{TaS}_{2}$, Nat. Commun. 11, 4215 (2020).

[7] B. Wang, Y. Liu, X. Luo, K. Ishigaki, K. Matsubayashi, W. Lu, Y. Sun, J. Cheng, and Y. Uwatoko, Universal phase diagram of superconductivity and charge density wave versus high hydrostatic pressure in pure and Se-doped $1 T-\mathrm{TaS}_{2}$, Phys. Rev. B 97, 220504(R) (2018).

[8] T. Ritschel, J. Trinckauf, G. Garbarino, M. Hanfland, M. v. Zimmermann, H. Berger, B. Büchner, and J. Geck Pressure dependence of the charge density wave in $1 T-\mathrm{TaS}_{2}$ and its relation to superconductivity, Phys. Rev. B 87, 125135 (2013).

[9] Q. Stahl, M. Kusch, F. Heinsch, G. Garbarino, N. Kretzschmar, K. Hanff, K. Rossnagel, J. Geck, and T. Ritschel, Collapse of layer dimerization in the photo-induced hidden state of $1 \mathrm{~T}-\mathrm{TaS}_{2}$, Nat. Commun. 11, 1247 (2020).

[10] B. Sipos, A. F. Kusmartseva, A. Akrap, H. Berger, L. Forró, and E. Tutis, From Mott state to superconductivity in $1 \mathrm{~T}-\mathrm{TaS}_{2}$, Nat. Mater. 7, 960 (2008).

[11] K. Sun, S. Sun, C. Zhu, H. Tian, H. Yang, and J. Li, Hidden CDW states and insulator-to-metal transition after a pulsed femtosecond laser excitation in layered chalcogenide $1 \mathrm{~T}-\mathrm{TaS}_{2-x} \mathrm{Se}_{x}$, Sci. Adv. 4, eaas9660 (2018).

[12] R. Ang, Z. C. Wang, C. L. Chen, J. Tang, N. Liu, Y. Liu, W. J. Lu, Y. P. Sun, T. Mori, and Y. Ikuhara, Atomistic origin of an ordered superstructure induced superconductivity in layered chalcogenides, Nat. Commun. 6, 6091 (2015).

[13] M. Klanjsek, A. Zorko, R. Zitko, J. Mravlje, Z. Jaglicic, P. K. Biswas, P. Prelovsek, D. Mihailovic, and D. Arcon, A hightemperature quantum spin liquid with polaron spins, Nat. Phys. 13, 1130 (2017).

[14] L. Ma, C. Ye, Y. Yu, X. F. Lu, X. Niu, S. Kim, D. Feng, D. Tománek, Y.-W. Son, X. H. Chen, and Y. Zhang, A metallic mosaic phase and the origin of Mott-insulating state in $1 \mathrm{~T}-\mathrm{TaS}_{2}$, Nat. Commun. 7, 10956 (2016).

[15] Y. Chen, W. Ruan, M. Wu, S. Tang, H. Ryu, H.-Z. Tsai, R. L. Lee, S. Kahn, F. Liou, C. Jia, O. R. Albertini, H. Xiong, T. Jia, Z. Liu, J. A. Sobota, A. Y. Liu, J. E. Moore, Z.-X. Shen, S. G. Louie, S.-K. Mo et al., Strong correlations and orbital texture in single-layer 1T-TaSe 2 , Nat. Phys. 16, 218 (2020).

[16] C. J. Butler, M. Yoshida, T. Hanaguri, and Y. Iwasa, Mottness versus unit-cell doubling as the driver of the insulating state in 1T-TaS 2 , Nat. Commun. 11, 2477 (2020).
[17] S. Qiao, X. Li, N. Wang, W. Ruan, C. Ye, P. Cai, Z. Hao, H. Yao, X. Chen, J. Wu, Y. Wang, and Z. Liu, Mottness Collapse in $1 \mathrm{~T}-\mathrm{TaS}_{2-x} \mathrm{Se}_{x}$ Transition-Metal Dichalcogenide: An Interplay between Localized and Itinerant Orbitals, Phys. Rev. X 7, 041054 (2017).

[18] L. Stojchevska, I. Vaskivskyi, T. Mertelj, P. Kusar, D. Svetin, S. Brazovskii, and D. Mihailovic, Ultrafast switching to a stable hidden quantum state in an electronic crystal, Science 344, 177 (2014).

[19] I. Vaskivskyi, J. Gospodaric, S. Brazovskii, D. Svetin, P. Sutar, E. Goreshnik, I. A. Mihailovic, T. Mertelj, and D. Mihailovic, Controlling the metal-to-insulator relaxation of the metastable hidden quantum state in $1 \mathrm{~T}-\mathrm{TaS}_{2}$, Sci. Adv. 1, e1500168 (2015).

[20] W. Wang, D. Dietzel, and A. Schirmeisen, Lattice discontinuities of $1 \mathrm{~T}-\mathrm{TaS}_{2}$ across first order charge density wave phase transitions, Sci. Rep. 9, 7066 (2019).

[21] C. Zhu, Y. Chen, F. Liu, S. Zheng, X. Li, A. Chaturvedi, J. Zhou, Q. Fu, Y. He, Q. Zeng, H. J. Fan, H. Zhang, W.-J. Liu, T. Yu, and Z. Liu, Light-tunable $1 \mathrm{~T}-\mathrm{TaS}_{2}$ charge-density-wave oscillators, ACS Nano 12, 11203 (2018).

[22] M. J. Trott and C. A. Hooley, Can Fermi surface nesting alone drive the charge-density-wave transition in monolayer vanadium diselenide? arXiv:2004.06665.

[23] A. V. Chubukov, M. Khodas, and R. M. Fernandes, Magnetism, Superconductivity, and Spontaneous Orbital Order in Iron-Based Superconductors: Which Comes First and Why? Phys. Rev. X 6, 041045 (2016).

[24] R. M. Fernandes and A. V. Chubukov, Low-energy microscopic models for iron-based superconductors: A review, Rep. Prog. Phys. 80, 014503 (2017).

[25] L. Fanfarillo, G. Giovannetti, M. Capone, and E. Bascones, Nematicity at the Hund's metal crossover in iron superconductors, Phys. Rev. B 95, 144511 (2017).

[26] S. Onari and H. Kontani, Self-consistent Vertex Correction Analysis for Iron-based Superconductors: Mechanism of Coulomb Interaction-Driven Orbital Fluctuations, Phys. Rev. Lett. 109, 137001 (2012).

[27] S. Onari, Y. Yamakawa, and H. Kontani, Sign-Reversing Orbital Polarization in the Nematic Phase of FeSe due to the $C_{2}$ Symmetry Breaking in the Self-Energy, Phys. Rev. Lett. 116, 227001 (2016).

[28] Y. Yamakawa, S. Onari, and H. Kontani, Nematicity and Magnetism in FeSe and Other Families of Fe-Based Superconductors, Phys. Rev. X 6, 021032 (2016).

[29] Y. Yamakawa and H. Kontani, Spin-Fluctuation-Driven Nematic Charge-Density Wave in Cuprate Superconductors: Impact of Aslamazov-Larkin Vertex Corrections, Phys. Rev. Lett. 114, 257001 (2015).

[30] M. Tsuchiizu, Y. Ohno, S. Onari and H. Kontani, Orbital Nematic Instability in the Two-Orbital Hubbard Model: Renormalization-Group+Constrained RPA Analysis, Phys. Rev. Lett. 111, 057003 (2013).

[31] R. Tazai, Y. Yamakawa, M. Tsuchiizu, and H. Kontani, Functional renormalization group study of orbital fluctuation mediated superconductivity: Impact of the electronboson coupling vertex corrections, Phys. Rev. B 94, 115155 (2016).

[32] M. Tsuchiizu, K. Kawaguchi, Y. Yamakawa, and H. Kontani, Multistage electronic nematic transitions in cuprate supercon- 
ductors: A functional-renormalization-group analysis, Phys. Rev. B 97, 165131 (2018).

[33] K. Kawaguchi, Y. Yamakawa, M. Tsuchiizu, and H. Kontani, Competing unconventional charge-density-wave states in cuprate superconductors: Spin-fluctuation-driven mechanism, J. Phys. Soc. Jpn. 86, 063707 (2017).

[34] R. Tazai and H. Kontani, Multipole fluctuation theory for heavy fermion systems: Application to multipole orders in $\mathrm{CeB}_{6}$, Phys. Rev. B 100, 241103(R) (2019).

[35] R. Tazai, Y. Yamakawa, M. Tsuchiizu, and H. Kontani, Prediction of pseudogap formation due to $d$-wave bondorder in organic superconductor $\kappa$-(BEDT-TTF) $)_{2}$ X, Phys. Rev. Research 3, L022014 (2021).

[36] R. Tazai, Y. Yamakawa, and H. Kontani, Emergence of charge loop current in the geometrically frustrated Hubbard model: A functional renormalization group study, Phys. Rev. B 103, L161112 (2021).

[37] R. Tazai, Y. Yamakawa, M. Tsuchiizu, and H. Kontani, $d$ - and $p$-wave quantum liquid crystal orders in cuprate superconductors, $\kappa$-(BEDT-TTF $)_{2} \mathrm{X}$, and coupled chain Hubbard models: functional-renormalization-group analysis, arXiv:2105.01872.

[38] H. Kontani, Y. Yamakawa, R. Tazai, and S. Onari, Odd-parity spin-loop-current order mediated by transverse spin fluctuations in cuprates and related electron systems, Phys. Rev. Research 3, 013127 (2021).

[39] S. Onari and H. Kontani, Hidden antiferronematic order in $\mathrm{Fe}-$ based superconductor $\mathrm{BaFe}_{2} \mathrm{As}_{2}$ and $\mathrm{NaFeAs}$ above $T_{S}$, Phys. Rev. Research 2, 042005(R) (2020); Origin of diverse nematic orders in Fe-based superconductors: $45^{\circ}$ rotated nematicity in $A \mathrm{Fe}_{2} \mathrm{As}_{2}(A=\mathrm{Cs}, \mathrm{Rb})$, Phys. Rev. B 100, 020507(R) (2019).

[40] M. Bovet, D. Popović, F. Clerc, C. Koitzsch, U. Probst, E. Bucher, H. Berger, D. Naumović, and P. Aebi, Pseudogapped Fermi surfaces of $1 T-\mathrm{TaS}_{2}$ and $1 T-\mathrm{TaSe}_{2}$ : A charge density wave effect, Phys. Rev. B 69, 125117 (2004).

[41] C. Sohrt, A. Stange, M. Bauer, and K. Rossnagel, How fast can a Peierls-Mott insulator be melted? Faraday Discuss. 171, 243 (2014).

[42] W. L. McMillan, Landau theory of charge-density waves in transition-metal dichalcogenides, Phys. Rev. B 12, 1187 (1975).

[43] K. Nakanishi and H. Shiba, Domain-like incommensurate charge-density-wave states and the first-order incommensuratecommensurate transitions in layered tantalum dichalcogenides. I. 1T-polytype, J. Phys. Soc. Jpn. 43, 1839 (1977).
[44] K. Nakanishi and H. Shiba, Theory of three-dimensional orderings of charge-density waves in $1 \mathrm{~T}-\mathrm{TaX}_{2}(\mathrm{X}: \mathrm{S}, \mathrm{Se})$, J. Phys. Soc. Jpn. 53, 1103 (1984).

[45] X.-L. Yu, D.-Y. Liu, Y.-M. Quan, J. Wu, H.-Q. Lin, K. Chang, and L.-J. Zou, Electronic correlation effects and orbital density wave in the layered compound $1 T-\mathrm{TaS}_{2}$, Phys. Rev. B 96, 125138 (2017).

[46] T. N. Ikeda, H. Tsunetsugu, and K. Yonemitsu, Photoinduced dynamics of commensurate charge density wave in $1 \mathrm{~T}-\mathrm{TaS}_{2}$ based on three-orbital Hubbard model, Appl. Sci. 9, 70 (2019).

[47] P. Darancet, A. J. Millis, and C. A. Marianetti, Threedimensional metallic and two-dimensional insulating behavior in octahedral tantalum dichalcogenides, Phys. Rev. B 90 , 045134 (2014).

[48] C. Chen, L. Su, A. H. Castro Neto, and V. M. Pereira, Discommensuration-driven superconductivity in the charge density wave phases of transition-metal dichalcogenides, Phys. Rev. B 99, 121108(R) (2019).

[49] T. Ritschel, H. Berger, and J. Geck, Stacking-driven gap formation in layered $1 T-\mathrm{TaS}_{2}$, Phys. Rev. B 98, 195134 (2018).

[50] L. F. Mattheiss, Band structures of transition-metaldichalcogenide layer compounds, Phys. Rev. B 8, 3719 (1973).

[51] See Supplemental Material at http://link.aps.org/supplemental/ 10.1103/PhysRevResearch.3.L032053 for model Hamiltonian, details of spin susceptibilities and form-factors, unfolded band spectrum, kernel function, and Ginzburg-Landau equations.

[52] H. Kontani, Anomalous transport phenomena in Fermi liquids with strong magnetic fluctuations, Rep. Prog. Phys. 71, 026501 (2008).

[53] H. Kontani, T. Saito, and S. Onari, Origin of orthorhombic transition, magnetic transition, and shear-modulus softening in iron pnictide superconductors: Analysis based on the orbital fluctuations theory, Phys. Rev. B 84, 024528 (2011).

[54] Y. Suzuki, T. Shimojima, T. Sonobe, A. Nakamura, M. Sakano, H. Tsuji, J. Omachi, K. Yoshioka, M. Kuwata-Gonokami, T. Watashige, R. Kobayashi, S. Kasahara, T. Shibauchi, Y. Matsuda, Y. Yamakawa, H. Kontani, and K. Ishizaka, Momentum-dependent sign inversion of orbital order in superconducting FeSe, Phys. Rev. B 92, 205117 (2015).

[55] W. Ku, T. Berlijn, and C.-C. Lee, Unfolding First-Principles Band Structures, Phys. Rev. Lett. 104, 216401 (2010). 